Supplement of Geosci. Model Dev., 10, 1587-1605, 2017

http://www.geosci-model-dev.net/10/1587/2017/

doi:10.5194/gmd-10-1587-2017-supplement

(C) Author(s) 2017. CC Attribution 3.0 License.

(c) (i)

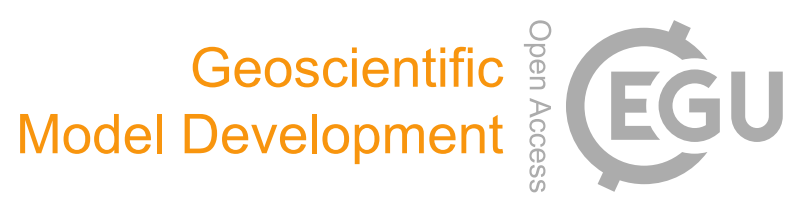

Supplement of

\title{
A framework for expanding aqueous chemistry in the Community Multiscale Air Quality (CMAQ) model version 5.1
}

Kathleen M. Fahey et al.

Correspondence to: Kathleen M. Fahey (fahey.kathleen@epa.gov)

The copyright of individual parts of the supplement might differ from the CC-BY 3.0 licence. 
Supplemental Information for "A framework for expanding aqueous chemistry in the Community Multiscale Air Quality (CMAQ) model version 5.1"

Table S1. Gas phase species that participate in phase transfer and associated gas phase diffusion and accommodation coefficients. 5 Unless otherwise indicated, Henry's Law coefficients are the base model's original values (from CMAQ subroutine hlconst.F).

\begin{tabular}{|c|c|c|c|c|}
\hline Reaction & $\mathrm{H}_{\text {law }}(\mathrm{M} / \mathbf{a t m})$ & $-\Delta \mathbf{H} / \mathbf{R}(\mathrm{K})$ & $\alpha^{a}$ & $D_{g}\left(\mathrm{~m}^{2} / \mathrm{s}\right) \times 10^{5 b}$ \\
\hline $\mathrm{SO}_{2 \text { (gas) }} \leftrightarrow \mathrm{SO}_{2 \text { (aq) }}$ & 1.4 & $2.9 \mathrm{E}+03$ & $1.10 \times 10^{-1}$ & 1.28 \\
\hline $\mathrm{HNO}_{3}$ (gas) $\leftrightarrow \mathrm{HNO}_{3}$ (aq) & $2.1 \mathrm{E}+05$ & $8.7 \mathrm{E}+03$ & $8.68 \times 10^{-2}$ & 1.32 \\
\hline $\mathrm{CO}_{2}$ (gas) $\leftrightarrow \mathrm{H}_{2} \mathrm{CO}_{3}$ (aq) & 3.6E-02 & $2.2 \mathrm{E}+03$ & $1.50 \times 10^{-4}$ & 1.55 \\
\hline $\mathrm{NH}_{3}$ (gas) $\leftrightarrow \mathrm{NH}_{4} \mathrm{OH}_{(\mathrm{aq})}$ & $6.1 \mathrm{E}+01$ & $4.2 \mathrm{E}+03$ & $9.10 \times 10^{-2}$ & 2.30 \\
\hline $\mathrm{H}_{2} \mathrm{O}_{2}$ (gas) $\leftrightarrow \mathrm{H}_{2} \mathrm{O}_{2}$ (aq) & $8.3 E+04$ & $7.4 \mathrm{E}+03$ & $1.53 \times 10^{-1}$ & 1.46 \\
\hline $\mathrm{O}_{3}$ (gas) $\leftrightarrow \mathrm{O}_{3}$ (aq) & $1.14 \mathrm{E}-02$ & $2.3 E+03$ & $1.00 \times 10^{-1}$ & 1.48 \\
\hline $\mathrm{HCOOH}_{(\mathrm{gas})} \leftrightarrow \mathrm{HCOOH}_{(\mathrm{aq})}$ & $8.9 \mathrm{E}+03$ & $6.1 \mathrm{E}+03$ & $2.29 \times 10^{-2}$ & 1.53 \\
\hline $\mathrm{MHP}_{\text {(gas) }} \leftrightarrow \mathrm{MHP}_{\text {(aq) }}$ & $3.1 \mathrm{E}+02$ & $5.2 \mathrm{E}+03$ & $6.76 \times 10^{-3}$ & 1.31 \\
\hline PAA $_{(\text {gas })} \leftrightarrow$ PAA $_{(\text {aq })}$ & $8.4 \mathrm{E}+02$ & $5.3 E+03$ & $1.90 \times 10^{-2}$ & 1.02 \\
\hline $\mathrm{HCl}_{\text {(gas) }} \leftrightarrow \mathrm{HCl}_{\text {(aq) }}$ & $1.9 \mathrm{E}+01$ & $6.0 \mathrm{E}+02$ & $1.16 \times 10^{-1}$ & 1.89 \\
\hline $\mathrm{GLY}_{\text {(gas) }} \leftrightarrow \mathrm{GLY}_{(\text {(aq) }}$ & $3.6 \mathrm{E}+05$ & & $2.30 \times 10^{-2 \mathrm{c}}$ & $1.15^{\mathrm{a}}$ \\
\hline MGLY $_{\text {(gas) }} \leftrightarrow$ MGLY $_{(\text {aq) }}$ & $3.2 \mathrm{E}+04^{\mathrm{e}}$ & & $2.30 \times 10^{-2 c}$ & $1.15^{\mathrm{d}}$ \\
\hline $\operatorname{IEPOX}_{\text {(gas) }} \leftrightarrow \operatorname{IEPOX}_{(\text {aq) }}$ & $2.7 \mathrm{E}+06^{\mathrm{f}}$ & & $2.00 \times 10^{-2 \mathrm{f}}$ & $1.00^{f}$ \\
\hline $\mathrm{MAE}_{\text {(gas) }} \leftrightarrow \mathrm{MAE}_{\text {(aq) }}$ & $1.2 \mathrm{E}+05^{\mathrm{f}}$ & & $2.00 \times 10^{-2 f}$ & $1.00^{f}$ \\
\hline $\mathrm{HMML}_{(\mathrm{gas})} \leftrightarrow \mathrm{HMML}_{(\mathrm{aq})}$ & $1.2 \mathrm{E}+05^{\mathrm{f}}$ & & $2.00 \times 10^{-2 f}$ & $1.00^{f}$ \\
\hline
\end{tabular}

${ }^{\circ} \mathrm{H}_{2} \mathrm{SO}_{4}$ is instantaneously transferred to aerosol $\mathrm{SO}_{4}$ and $\mathrm{N}_{2} \mathrm{O}_{5}$ to $\mathrm{HNO}_{3}$ (gas) at the start of cloud processing. ${ }^{a}$ CAPRAM 2.4 tables (http://projects.tropos.de/capram/capram_24.html)*. bHerrmann et al. (2000)*. 'Lim et al. (2005)*. ${ }^{\text {d} A s s u m e d ~ e q u a l ~ t o ~ D g ~ f o r ~ g l y o x a l . ~}{ }^{\mathrm{e}}$ The Henry's Law coefficient for methylglyoxal is replaced with that of glyoxal when the CB05 gas phase mechanism is used (Carlton et al., 2010). fPye et al. (2013). *and the original sources therein

Table S2. Dissociation reactions and equilibrium constants. Ionic species are considered explicitly and dissociation equilibrium reactions are described as a set of forward and backward reactions. Activity coefficients are rolled into the forward and backward reaction rates.

\begin{tabular}{|c|c|c|c|}
\hline Reaction & $K_{\text {eq,298 }}{ }^{a}[M]$ & $-\Delta \mathbf{E}_{\mathbf{a}} / \mathbf{R}[\mathbf{K}]^{\#}$ & $k_{b}{ }^{a, d}\left[M^{-1} s^{-1}\right]$ \\
\hline $\mathrm{SO}_{2} \leftrightarrow \mathrm{HSO}_{3}^{-}+\mathrm{H}^{+}$ & $1.39 \times 10^{-2}$ & $1.87 \times 10^{3}$ & $2.0 \times 10^{8}$ \\
\hline $\mathrm{HSO}_{3}^{-} \leftrightarrow \mathrm{SO}_{3}^{2-}+\mathrm{H}^{+}$ & $6.72 \times 10^{-8}$ & $3.55 \times 10^{2}$ & $5.0 \times 10^{10}$ \\
\hline $\mathrm{HNO}_{3} \leftrightarrow \mathrm{NO}_{3}^{-}+\mathrm{H}^{+}$ & $1.7 \times 10^{1}$ & & $5.0 \times 10^{10}$ \\
\hline $\mathrm{NH}_{4} \mathrm{OH} \leftrightarrow \mathrm{NH}_{4}^{+}+\mathrm{OH}^{-}$ & $1.77 \times 10^{-5}$ & $-7.10 \times 10^{2}$ & $3.4 \times 10^{10}$ \\
\hline $\mathrm{H}_{2} \mathrm{CO}_{3} \leftrightarrow \mathrm{HCO}_{3}^{-}+\mathrm{H}^{+}$ & $4.3 \times 10^{-7}$ & $-9.95 \times 10^{2}$ & $6.4 \times 10^{4}$ \\
\hline $\mathrm{HCO}_{3}^{-} \leftrightarrow \mathrm{CO}_{3}^{2-}+\mathrm{H}^{+}$ & $4.68 \times 10^{-11}$ & $-1.79 \times 10^{3}$ & $5.0 \times 10^{10}$ \\
\hline $\mathrm{HCOOH} \leftrightarrow \mathrm{HCOO}^{-}+\mathrm{H}^{+}$ & $1.8 \times 10^{-4} \mathrm{~b}$ & $-2.00 \times 10^{1}$ & $5.0 \times 10^{10}$ \\
\hline $\mathrm{HCl} \leftrightarrow \mathrm{Cl}^{-}+\mathrm{H}^{+}$ & $1.74 \times 10^{6 b}$ & $6.90 \times 10^{3}$ & $5.0 \times 10^{10}$ \\
\hline $\mathrm{H}_{2} \mathrm{O} \leftrightarrow \mathrm{OH}^{-}+\mathrm{H}^{+}$ & $1.8 \times 10^{-16 c}$ & $-6.95 \times 10^{3}$ & $1.4 \times 10^{11}$ \\
\hline $\mathrm{H}_{2} \mathrm{SO}_{4} \leftrightarrow \mathrm{HSO}_{4}^{-}+\mathrm{H}^{+}$ & $1.0 \times 10^{3 \mathrm{~b}}$ & & $5.0 \times 10^{10}$ \\
\hline $\mathrm{HSO}_{4}^{-} \leftrightarrow \mathrm{SO}_{4}^{2-}+\mathrm{H}^{+}$ & $1.02 \times 10^{-2}$ & $2.45 \times 10^{3}$ & $1.0 \times 10^{11}$ \\
\hline
\end{tabular}

${ }^{\mathrm{a}}$ Warneck and Williams (2012)*. ${ }^{\mathrm{b}}$ Seinfeld and Pandis(2006)*. ${ }^{\mathrm{C}}$ Removed $\left[\mathrm{H}_{2} \mathrm{O}\right]$ concentration of $55.5 \mathrm{M}$ from the original equilibrium constant. ${ }^{\mathrm{d}}$ Graedel and Weschler (1981)*. *and the original sources therein

$\mathrm{kf}_{\mathrm{f}}=\mathrm{Keq} \mathrm{i}, \mathrm{T} \mathrm{x} \mathrm{kb}$ " Note that while there are some minor differences between some of the equilibrium constants used here compared to AQCHEM, box model tests indicate that these small differences only have a minimal impact on predicted concentrations 
Table S3. Chemical kinetic reactions.

\begin{tabular}{|c|c|c|c|}
\hline Reaction & \multicolumn{2}{|c|}{$\begin{array}{l}\text { Rate coefficient in appropriate units of } \\
\text { M,s }(298 K) ;-E_{a} / R(K)^{\$}\end{array}$} & Notes \\
\hline $\mathrm{HSO}_{3}{ }^{-}+\mathrm{H}_{2} \mathrm{O}_{2}+\mathrm{H}^{+} \rightarrow \mathrm{SO}_{4}{ }^{2-}+2 \mathrm{H}^{+}$ & \multicolumn{2}{|c|}{$7.45 \mathrm{E}+7 /(1+13[\mathrm{H}+]) ;-4756.08$} & \\
\hline $\mathrm{SO}_{2}+\mathrm{O}_{3} \rightarrow \mathrm{SO}_{4}^{2-}+2 \mathrm{H}^{+}$ & \multicolumn{2}{|l|}{$2.4 \mathrm{E}+4 ; 0.0$} & Q \\
\hline $\mathrm{HSO}_{3}{ }^{-}+\mathrm{O}_{3} \rightarrow \mathrm{SO}_{4}{ }^{2-}+\mathrm{H}^{+}$ & \multicolumn{2}{|c|}{$3.7 \mathrm{E}+5 ;-5530.88$} & $\mathrm{Q}$ \\
\hline $\mathrm{SO}_{3}^{2-}+\mathrm{O}_{3} \rightarrow \mathrm{SO}_{4}^{2-}$ & \multicolumn{2}{|c|}{$1.5 \mathrm{E}+9 ;-5280.56$} & Q \\
\hline $\mathrm{HSO}_{3}{ }^{-}+\mathrm{MHP}+\mathrm{H}^{+} \rightarrow \mathrm{SO}_{4}{ }^{2-}+2 \mathrm{H}^{+}$ & \multicolumn{2}{|c|}{$1.9 \mathrm{E}+7 ;-3799.5$} & \\
\hline${ }^{*} \mathrm{HSO}_{3}{ }^{-}+\mathrm{PAA}+\mathrm{H}^{+} \rightarrow \mathrm{SO}_{4}{ }^{2-}+2 \mathrm{H}^{+}$ & \multicolumn{2}{|c|}{$3.6 \mathrm{E}+7 ;-3999.2$} & * \\
\hline${ }^{*} \mathrm{HSO}_{3}{ }^{-}+\mathrm{PAA} \rightarrow \mathrm{SO}_{4}{ }^{2-}+\mathrm{H}^{+}$ & \multicolumn{2}{|l|}{$7 \mathrm{E}+2 ; 0.0$} & \\
\hline $\mathrm{SO}_{2}(+\mathrm{Mn}(\mathrm{II})) \rightarrow \mathrm{SO}_{4}^{2-}+2 \mathrm{H}^{+}$ & \multicolumn{2}{|l|}{$7.5 \mathrm{E}+2 ; 0.0$} & \# \\
\hline $\mathrm{HSO}_{3}{ }^{-}(+\mathrm{Mn}(\mathrm{II})) \rightarrow \mathrm{SO}_{4}{ }^{2-}+\mathrm{H}^{+}$ & \multicolumn{2}{|l|}{$7.5 \mathrm{E}+2 ; 0.0$} & \# \\
\hline $\mathrm{SO}_{3}{ }^{2-}(+\mathrm{Mn}(\mathrm{II})) \rightarrow \mathrm{SO}_{4}{ }^{2-}$ & \multicolumn{2}{|l|}{$7.5 \mathrm{E}+2 ; 0.0$} & $\#$ \\
\hline $\mathrm{SO}_{2}(+\mathrm{Fe}(\mathrm{III})) \rightarrow \mathrm{SO}_{4}^{2-}+2 \mathrm{H}^{+}$ & \multicolumn{2}{|l|}{$2.6 \mathrm{E}+3 ; 0.0$} & \# \\
\hline $\mathrm{HSO}_{3}{ }^{-}(+\mathrm{Fe}(\mathrm{III})) \rightarrow \mathrm{SO}_{4}^{2-}+\mathrm{H}^{+}$ & \multicolumn{2}{|l|}{$2.6 \mathrm{E}+3 ; 0.0$} & $\#$ \\
\hline $\mathrm{SO}_{3}{ }^{2-}(+\mathrm{Fe}(\mathrm{III})) \rightarrow \mathrm{SO}_{4}{ }^{2-}$ & \multicolumn{2}{|l|}{$2.6 \mathrm{E}+3 ; 0.0$} & $\#$ \\
\hline $\mathrm{SO}_{2}(+\mathrm{Mn}(\mathrm{II})+\mathrm{Fe}(\mathrm{III})) \rightarrow \mathrm{SO}_{4}^{2-}+2 \mathrm{H}^{+}$ & \multicolumn{2}{|l|}{$1 \mathrm{E}+10 ; 0.0$} & \# \\
\hline $\mathrm{HSO}_{3}{ }^{-}(+\mathrm{Mn}(\mathrm{II})+\mathrm{Fe}(\mathrm{III})) \rightarrow \mathrm{SO}_{4}{ }^{2-}+\mathrm{H}^{+}$ & \multicolumn{2}{|l|}{$1 \mathrm{E}+10 ; 0.0$} & $\#$ \\
\hline $\mathrm{SO}_{3}{ }^{2-}(+\mathrm{Mn}(\mathrm{II})+\mathrm{Fe}(\mathrm{III})) \rightarrow \mathrm{SO}_{4}{ }^{2-}$ & \multicolumn{2}{|l|}{$1 \mathrm{E}+10 ; 0.0$} & $\#$ \\
\hline $\mathrm{GLY}+\mathrm{OH} \bullet \rightarrow 0.04 \mathrm{ORGC}$ & \multicolumn{2}{|l|}{$3 E+10 ; 0.0$} & Fixed $\mathrm{OH} \bullet$ \\
\hline \multirow[t]{2}{*}{$\mathrm{MGLY}+\mathrm{OH} \bullet \rightarrow 0.04 \mathrm{ORGC}$} & \multicolumn{2}{|l|}{$3 E+10 ; 0.0$} & Fixed $\mathrm{OH} \bullet$ \\
\hline & $\mathrm{k}_{1}\left(\mathrm{M}^{-2} \mathrm{~s}^{-1}\right)$ & $\mathrm{k}_{2}\left(\mathrm{M}^{-2} \mathrm{~s}^{-1}\right)$ & \\
\hline IEPOX $+\mathrm{H}_{2} \mathrm{O} \rightarrow$ 2-methyltetrol & $9 \mathrm{E}-04$ & $1.3 \mathrm{E}-05$ & Rate $=$ KIEPOX $*$ Parent \\
\hline $\mathrm{IEPOX}+\mathrm{SO}_{4}{ }^{2-} \rightarrow$ IEPOX_OS & 2E-04 & 2.9E-06 & Hydrocarbon * \\
\hline $\mathrm{IEPOX}+\mathrm{NO}_{3}{ }^{-} \rightarrow$ IEPOX_ON ${ }^{\S}$ & $2 \mathrm{E}-04$ & 2.9E-06 & Nucleophile * Q \\
\hline IEPOX + 2-methyltetrol $\rightarrow$ dimers & 2E-04 & 2.9E-06 & \\
\hline IEPOX + IEPOX_OS $\rightarrow$ dimers & 2E-04 & 2.9E-06 & whe \\
\hline IEPOX + IEPOX_ON $\rightarrow$ dimers $^{\S}$ & 2E-04 & 2.9E-06 & KIEPOX $=\mathrm{k}_{1} *\left[\mathrm{H}^{+}\right]+$ \\
\hline $\mathrm{MAE}+\mathrm{H}_{2} \mathrm{O} \rightarrow 2-\mathrm{MG}$ & $9 \mathrm{E}-04$ & 1.3E-05 & $\mathrm{k}_{2} *\left[\mathrm{HSO}^{-}\right]$ \\
\hline $\mathrm{MAE}+\mathrm{SO}_{4}{ }^{2-} \rightarrow$ MPAN_OS & 2E-04 & 2.9E-06 & \\
\hline $\mathrm{MAE}+\mathrm{NO}_{3}{ }^{-} \rightarrow$ MPAN_ON ${ }^{\S}$ & 2E-04 & 2.9E-06 & \\
\hline $\mathrm{MAE}+2-\mathrm{MG} \rightarrow$ dimers $^{\S}$ & 2E-04 & 2.9E-06 & $\mathrm{H}_{2} \mathrm{O}$ concentration \\
\hline $\mathrm{MAE}+\mathrm{MPAN} \_\mathrm{OS} \rightarrow$ dimers $^{\S}$ & 2E-04 & 2.9E-06 & remains fixed (55.5 M) \\
\hline $\mathrm{MAE}+$ MPAN_ON $\rightarrow$ dimers $^{\S}$ & 2E-04 & 2.9E-06 & \\
\hline $\mathrm{HMML}+\mathrm{H}_{2} \mathrm{O} \rightarrow 2-\mathrm{MG}$ & $9 \mathrm{E}-04$ & $1.3 \mathrm{E}-05$ & \\
\hline $\mathrm{HMML}+\mathrm{SO}_{4}{ }^{2-} \rightarrow$ MPAN_OS & 2E-04 & 2.9E-06 & \\
\hline $\mathrm{HMML}+\mathrm{NO}_{3}{ }^{-} \rightarrow$ MPAN_ON ${ }^{\S}$ & 2E-04 & 2.9E-06 & \\
\hline HMML $+2-\mathrm{MG} \rightarrow$ dimers $^{\S}$ & 2E-04 & 2.9E-06 & \\
\hline HMML + MPAN_OS $\rightarrow$ dimers $^{\S}$ & $2 \mathrm{E}-04$ & 2.9E-06 & \\
\hline HMML + MPAN_ON $\rightarrow$ dimers $\S$ & 2E-04 & 2.9E-06 & \\
\hline
\end{tabular}

${ }^{\$}$ Rate coefficients are equal to those used in the standard version of AQCHEM. Q Aqueous diffusion correction factor applied; *included here as two reactions to accommodate the original single rate expression which included $\mathrm{pH}$ dependent and pH independent elements. ${ }^{*} \mathrm{~A}$ $\mathrm{S}(\mathrm{VI})$ inhibition factor of the following form is applied to these reaction rates: $\left(1+75[\mathrm{~S}(\mathrm{VI})]^{0.67}\right)^{-1}$. (Species in parentheses on the left hand

5 side of the rate expression indicate species that affect the reaction rate calculation but whose concentrations are not consumed in the reaction.) §Included here but not included in the version of AQCHEM-KMTI released with CMAQv5.1 
Table S4. CMAQ species and local AQCHEM-KMT(I) species. The “G”, “L”, “A”, and "WD” prefixes in column 1 refer to the gas-phase, aqueous-phase, Aitken aerosol, and wet deposition portion of the species respectively. Shaded cells include species specific to AQCHEM-KMTI. CMAQ aerosol concentration and wet deposition indices are of the form: [species, mode].

\begin{tabular}{|c|c|c|}
\hline $\begin{array}{l}\text { AQCHEM-KMT(I) local dynamic } \\
\text { species }\end{array}$ & CMAQ species & Name and Notes \\
\hline G_SO2, L_SO2, WD_SO2 & GAS(LSO2), GASWDEP(LSO2) & Sulfur dioxide \\
\hline G_HNO3, L_HNO3, WD_HNO3 & $\begin{array}{l}\text { GAS(LHNO3)/AEROSOL(LNO3, 2/3), } \\
\text { GASWDEP(LHNO3) }\end{array}$ & Nitric acid, nitrate \\
\hline G_CO2, L_H2CO3, WD_CO2 & GAS(LCO2), GASWDEP(LCO2) & Carbon dioxide \\
\hline G_NH3, L_NH4OH, WD_NH4OH & $\begin{array}{l}\text { GAS(LNH3), AEROSOL(LNH4, 2/3), } \\
\text { GASWDEP(LNH3) }\end{array}$ & Ammonia, ammonium \\
\hline G_H2O2, L_H2O2, WD_H2O2 & GAS(LH2O2), GASWDEP(LH2O2) & Hydrogen peroxide \\
\hline G_O3, L_O3, WD_O3 & GAS(LO3), GASWDEP(LO3) & Ozone \\
\hline $\begin{array}{l}\text { G_HCOOH, L_HCOOH, } \\
\text { WD_HCOOH }\end{array}$ & GAS(LFOA), GASWDEP(LFOA) & Formic acid \\
\hline G_MHP, L_MHP, WD_MHP & GAS(LMHP), GASWDEP(LMHP) & $\begin{array}{l}\text { Methylhydroxyperoxide } \\
\text { (CH3OOH) }\end{array}$ \\
\hline G_PAA, L_PAA, WD_PAA & GAS(LPAA), GASWDEP(LPAA) & $\begin{array}{l}\text { peroxyacetic acid } \\
(\mathrm{CH} 3 \mathrm{C}(\mathrm{O}) \mathrm{OOH})\end{array}$ \\
\hline G_HCL, L_HCL, WD_HCL & GAS(HCL), GASWDEP(HCL) & Hydrochloric acid \\
\hline G_GLY, L_GLY, WD_GLY & GAS(LGLY), GASWDEP(LGLY) & Glyoxal \\
\hline G_MGLY, L_MGLY, WD_MGLY & GAS(LMGLY), GASWDEP(LMGLY) & Methylglyoxal \\
\hline A_NO3AKN & AEROSOL(LNO3,1) & Aitken nitrate \\
\hline A_NH4AKN & AEROSOL(LNH4,1) & Aitken ammonium \\
\hline A_CLAKN & AEROSOL(LCL,1) & Aitken chloride \\
\hline A_NAAKN & AEROSOL(LNA,1) & Aitken sodium \\
\hline A_SO4AKN & AEROSOL(LSO4,1) & Aitken sulfate \\
\hline A_PECAKN & AEROSOL(LEC,1) & Aitken elemental carbon \\
\hline A_POAAKN & AEROSOL(LPOA,1) & Aitken primary OA \\
\hline A_PRIAKN & AEROSOL(LPRI,1) & Aitken other primary \\
\hline L_SO4MIN2, WD_H2SO4 & $\begin{array}{l}\text { AEROSOL(LSO4, 2/3), } \\
\text { AERWDEP(LSO4, 2/3) }\end{array}$ & Sulfate \\
\hline L_NO3MIN, WD_NO3MIN & $\begin{array}{l}\text { GAS(LHNO3), AEROSOL(LNO3, 2/3), } \\
\text { AERWDEP(LNO3, 2/3) }\end{array}$ & Nitrate \\
\hline L_NH4PLUS, WD_NH4PLUS & $\begin{array}{l}\text { GAS(LNH3), AEROSOL(LNH4, } 2 \text { and } \\
\text { 3), AERWDEP(LNH4, 2/3) }\end{array}$ & Ammonium \\
\hline L_CLMIN, WD_CLMIN & $\begin{array}{l}\text { AEROSOL(LCL, 2/3), AERWDEP(LCL, } \\
2 / 3)\end{array}$ & Chloride \\
\hline L_PRIACC, WD_PRIACC & $\begin{array}{l}\text { AEROSOL(LPRI, 2), AERWDEP(LPRI, } \\
\text { 2) }\end{array}$ & Other primary \\
\hline L_PECACC, WD_PECACC & $\begin{array}{l}\text { AEROSOL(LEC, 2), AERWDEP(LEC, } \\
\text { 2) }\end{array}$ & Elemental carbon \\
\hline L_ORGC, WD_ORGC & $\begin{array}{l}\text { AEROSOL(LORGC, 2), } \\
\text { AERWDEP(LORGC, 2) }\end{array}$ & Cloud SOA \\
\hline L_POAACC, WD_POAACC & $\begin{array}{l}\text { AEROSOL(LPOA, 2), } \\
\text { AERWDEP(LPOA, 2) }\end{array}$ & Primary OA \\
\hline L_HPLUS, WD_HPLUS & HPWDEP & $\mathrm{H}^{+}$ \\
\hline L_OHMIN & -- & $\mathrm{OH}^{-}$ \\
\hline
\end{tabular}




\begin{tabular}{|c|c|c|}
\hline L_FEPLUS3, WD_FEPLUS3\% & $\begin{array}{l}\text { AEROSOL(LFEACC, 2), } \\
\text { AERWDEP(LFEACC, 2), } \\
\text { AEROSOL(LSOILC, 3), } \\
\text { AERWDEP(LSOILC, 3), } \\
\text { AEROSOL(LANTHC, 3), } \\
\text { AERWDEP(LANTHC, 3) }\end{array}$ & $\begin{array}{l}\text { Soluble iron }\left({ }^{3+}\right) ;{ }^{\%} \mathrm{AQCHEM} \\
\text { species include contributions } \\
\text { from coarse aerosol }\end{array}$ \\
\hline L_MNPLUS2, WD_MNPLUS2 ${ }^{\%}$ & $\begin{array}{l}\text { AEROSOL(LMNACC, 2), } \\
\text { AERWDEP(LMNACC, 2), } \\
\text { AEROSOL(LSOILC, 3), } \\
\text { AERWDEP(LSOILC, 3), } \\
\text { AEROSOL(LANTHC, 3), } \\
\text { AERWDEP(LANTHC, 3) }\end{array}$ & $\begin{array}{l}\text { Soluble manganese }\left({ }^{2+}\right) \text {; } \\
{ }^{\%} \text { AQCHEM species include } \\
\text { contributions from coarse } \\
\text { aerosol }\end{array}$ \\
\hline L_HSO3MIN, (WD_SO2) & GAS(LSO2) & Bisulfite \\
\hline L_SO3MIN2, (WD_SO2) & GAS(LSO2) & Sulfite \\
\hline L_HCO3MIN, (WD_CO2) & GAS(LCO2) & Bicarbonate \\
\hline L_CO3MIN2, (WD_CO2) & GAS(LCO2) & Carbonate \\
\hline L_HCOOMIN, (WD_HCOOH) & GAS(LFOA) & Formate \\
\hline L_HSO4MIN, (WD_H2SO4) & AEROSOL(LSO4, 2/3) & Bisulfate \\
\hline L_CAPLUS2, WD_CAPLUS2 $\%$ & $\begin{array}{l}\text { AEROSOL(LCAACC, 2), } \\
\text { AERWDEP(LCAACC, 2), } \\
\text { AEROSOL(LSOILC, 3), } \\
\text { AERWDEP(LSOILC, 3), } \\
\text { AEROSOL(LANTHC, 3), } \\
\text { AERWDEP(LANTHC, 3), } \\
\text { AEROSOL(LSEASC, 3), } \\
\text { AERWDEP(LSEASC, 3) }\end{array}$ & $\begin{array}{l}\text { Calcium }\left({ }^{2+}\right) ;{ }^{\%} \text { AQCHEM } \\
\text { species include contributions } \\
\text { from coarse aerosol }\end{array}$ \\
\hline L_MGPLUS2, WD_MGPLUS2 ${ }^{\%}$ & $\begin{array}{l}\text { AEROSOL(LMGACC, 2), } \\
\text { AERWDEP(LMGACC, 2), } \\
\text { AEROSOL(LSOILC, 3), } \\
\text { AERWDEP(LSOILC, 3), } \\
\text { AEROSOL(LANTHC, 3), } \\
\text { AERWDEP(LANTHC, 3), } \\
\text { AEROSOL(LSEASC, 3), } \\
\text { AERWDEP(LSEASC, 3) }\end{array}$ & $\begin{array}{l}\text { Magnesium }\left({ }^{2+}\right) ;{ }^{\%} \text { AQCHEM } \\
\text { species include contributions } \\
\text { from coarse aerosol }\end{array}$ \\
\hline L_KPLUS, WD_KPLUS $\%$ & $\begin{array}{l}\text { AEROSOL(LKACC, 2), } \\
\text { AERWDEP(LKACC, 2), } \\
\text { AEROSOL(LSOILC, 3), } \\
\text { AERWDEP(LSOILC, 3), } \\
\text { AEROSOL(LANTHC, 3), } \\
\text { AERWDEP(LANTHC, 3), } \\
\text { AEROSOL(LSEASC, 3), } \\
\text { AERWDEP(LSEASC, 3) }\end{array}$ & $\begin{array}{l}\text { Potassium }\left({ }^{+}\right) ;{ }^{\%} \text { AQCHEM } \\
\text { species include contributions } \\
\text { from coarse aerosol }\end{array}$ \\
\hline L_NAPLUS, WD_NAPLUS $\%$ & $\begin{array}{l}\text { AEROSOL(LNA, 2), AERWDEP(LNA, } \\
\text { 2), AEROSOL(LSOILC, 3), } \\
\text { AERWDEP(LSOILC, 3), } \\
\text { AEROSOL(LANTHC, 3), } \\
\text { AERWDEP(LANTHC, 3), } \\
\text { AEROSOL(LSEASC, 3), } \\
\text { AERWDEP(LSEASC, 3) }\end{array}$ & $\begin{array}{l}\text { Sodium }\left({ }^{+}\right) ;{ }^{\%} \text { AQCHEM } \\
\text { species include contributions } \\
\text { from coarse aerosol }\end{array}$ \\
\hline G_IEPOX, L_IEPOX, WD_IEPOX & GAS(LIEPOX), GASWDEP(LIEPOX) & Isoprene epoxydiols \\
\hline
\end{tabular}




\begin{tabular}{|c|c|c|}
\hline G_IMAE, L_IMAE, WD_IMAE & GAS(LIMAE), GASWDEP(LIMAE) & Methacrylic acid epoxide \\
\hline $\begin{array}{l}\text { G_IHMML, L_IHMML, } \\
\text { WD_IHMML }\end{array}$ & GAS(LIHMML), GASWDEP(LIHMML) & $\begin{array}{l}\text { Hydroxymethylmethyl- } \alpha \text { - } \\
\text { lactone }\end{array}$ \\
\hline L_IETET, WD_IETET & $\begin{array}{l}\text { AEROSOL(LIETET, 2), } \\
\text { AERWDEP(LIETET, 2) }\end{array}$ & 2-methyltetrols \\
\hline L_IEOS, WD_IEOS & $\begin{array}{l}\text { AEROSOL(LIEOS, 2), } \\
\text { AERWDEP(LIEOS, 2) }\end{array}$ & $\begin{array}{l}\text { IEPOX-derived } \\
\text { organosulfate }\end{array}$ \\
\hline L_IEON, WD_IEON ${ }^{* *}$ & $\begin{array}{l}\text { AEROSOL(LIEON, 2), } \\
\text { AERWDEP(LIEON, 2) }\end{array}$ & $\begin{array}{l}\text { IEPOX-derived } \\
\text { organonitrate. }{ }^{* *} \text { not included } \\
\text { in CMAQv5.1 but used here }\end{array}$ \\
\hline L_DIMER, WD_DIMER & $\begin{array}{l}\text { AEROSOL(LDIMER, 2), } \\
\text { AERWDEP(LDIMER, 2) }\end{array}$ & dimers \\
\hline L_IMGA, WD_IMGA & $\begin{array}{l}\text { AEROSOL(LIMGA, 2), } \\
\text { AERWDEP(LIMGA, 2) }\end{array}$ & 2-methylglyceric acid \\
\hline L_IMOS, WD_IMOS & $\begin{array}{l}\text { AEROSOL(LIMOS, 2), } \\
\text { AERWDEP(LIMOS, 2) }\end{array}$ & MPAN-derived organosulfate \\
\hline L_IMON, WD_IMON** & $\begin{array}{l}\text { AEROSOL(LIMON, 2), } \\
\text { AERWDEP(LIMON, 2) }\end{array}$ & $\begin{array}{l}\text { MPAN-derived } \\
\text { organonitrate. }{ }^{* *} \text { not included } \\
\text { in CMAQv5.1 but used here }\end{array}$ \\
\hline G_OH, L_OH* & GAS(LHO) & $\begin{array}{l}\text { Hydroxyl radical. * Mapped in } \\
\text { from CMAQ but kept } \\
\text { constant during/after cloud } \\
\text { processing }\end{array}$ \\
\hline
\end{tabular}

Table S5. Box model test scenarios. Initial aerosol species concentrations are held constant for the variable scenarios and are associated with an initial droplet $\mathrm{pH}$ of $\sim 5$. Default gas concentrations were set at $1 \mathrm{ppb}$ and modal aerosol species concentrations were set to $0.1 \mu \mathrm{g} / \mathrm{m}^{3}$, with the following exceptions: $\mathrm{O}_{3}=50 \mathrm{ppb}, \mathrm{CO}_{2}=340 \mathrm{ppm}, \mathrm{H}_{2} \mathrm{SO}_{4}=0.1 \mathrm{ppb} \mathrm{N}_{2} \mathrm{O}_{5}=0.1 \mathrm{ppb}, \mathrm{SO}_{4}{ }^{2-}=4.2$ $\mu \mathrm{g} / \mathrm{m}^{3}$, Primary inorganic (inert) $=2.1 \mu \mathrm{g} / \mathrm{m}^{3}$, Primary OA $=1.1 \mu \mathrm{g} / \mathrm{m}^{3}, \mathrm{SOA}=2.1 \mu \mathrm{g} / \mathrm{m}^{3}, \mathrm{EC}=1.1 \mu \mathrm{g} / \mathrm{m}^{3}, \mathrm{Cl}^{-}=2.3 \mu \mathrm{g} / \mathrm{m}^{3}, \mathrm{NH}{ }^{+}=$ $2.2 \mu \mathrm{g} / \mathrm{m}^{3}, \mathrm{NO}_{3}{ }^{-}=4.1 \mu \mathrm{g} / \mathrm{m}^{3}$, Na, accumulation mode Fe, $\mathrm{Mn}, \mathrm{Ca}=0.3 \mu \mathrm{g} / \mathrm{m}^{3}$, coarse soil and anthropogenic coarse $=2 \mu \mathrm{g} / \mathrm{m}^{3}$, coarse sea salt cations $=1 \mu \mathrm{g} / \mathrm{m}^{3}$, Pressure $=1 \mathrm{~atm}$.

\begin{tabular}{|l|l|}
\hline Variable input & Values \\
\hline Liquid Water content $\left(\mathrm{g} / \mathrm{m}^{3}\right)$ & $0.05,0.1,0.2,0.3$ \\
\hline Precipitation rate $(\mathrm{mm} / \mathrm{hr})$ & 0,2 \\
\hline Temperature $(\mathrm{K})$ & 270,283 \\
\hline Daylight (binary) & 0,1 \\
\hline Integration time $(\mathrm{s})$ & $60,300,720$ \\
\hline $\mathrm{SO}_{2}(\mathrm{ppb})$ & $1,5,10$ \\
\hline $\mathrm{H}_{2} \mathrm{O}_{2}(\mathrm{ppb})$ & $0.1,1,5$ \\
\hline$(\mathrm{M}) \mathrm{GLY}(\mathrm{ppb})$ & $0.5,2$ \\
\hline $\mathrm{OH}(\mathrm{ppb})$ & $10^{-3}, 10^{-5}, 10^{-7}$ \\
\hline $\mathrm{NH}_{3}(\mathrm{ppb})$ & 1,5 \\
\hline $\mathrm{HNO}_{3}(\mathrm{ppb})$ & 1,5 \\
\hline
\end{tabular}


CLOUD SOA (ORGC) $\left(\mu \mathrm{g} / \mathrm{m}^{3}\right)$ - Differences due to timestepping versus

kinetic mass transfer

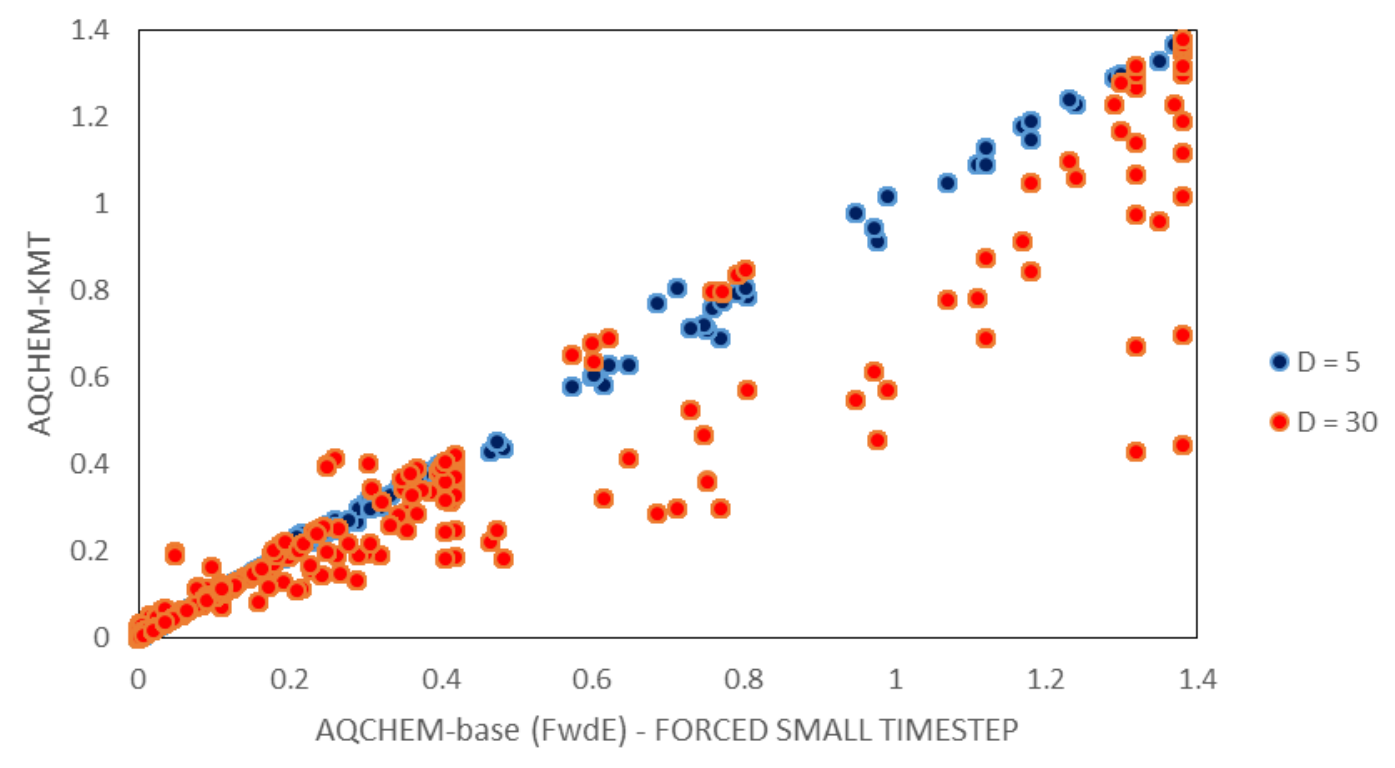

Figure S1: Cloud SOA (ORGC) predicted by AQCHEM-KMT at 5 (blue) and $30 \mu \mathrm{m}$ (red) droplet diameters versus standard AQCHEM with a forced small time step. Compared to Figure 2 where AQCHEM is run with its standard time-stepping procedure, it appears that the differences between the models for ORGC are driven by both mass transfer limitations as well as the time5 stepping procedure (which in standard AQCHEM is based on sulfate production only). 


\section{Total $\mathrm{SO}_{4}\left(\mu \mathrm{g} / \mathrm{m}^{3}\right)$ - Effect of initial droplet $\mathrm{pH}$}

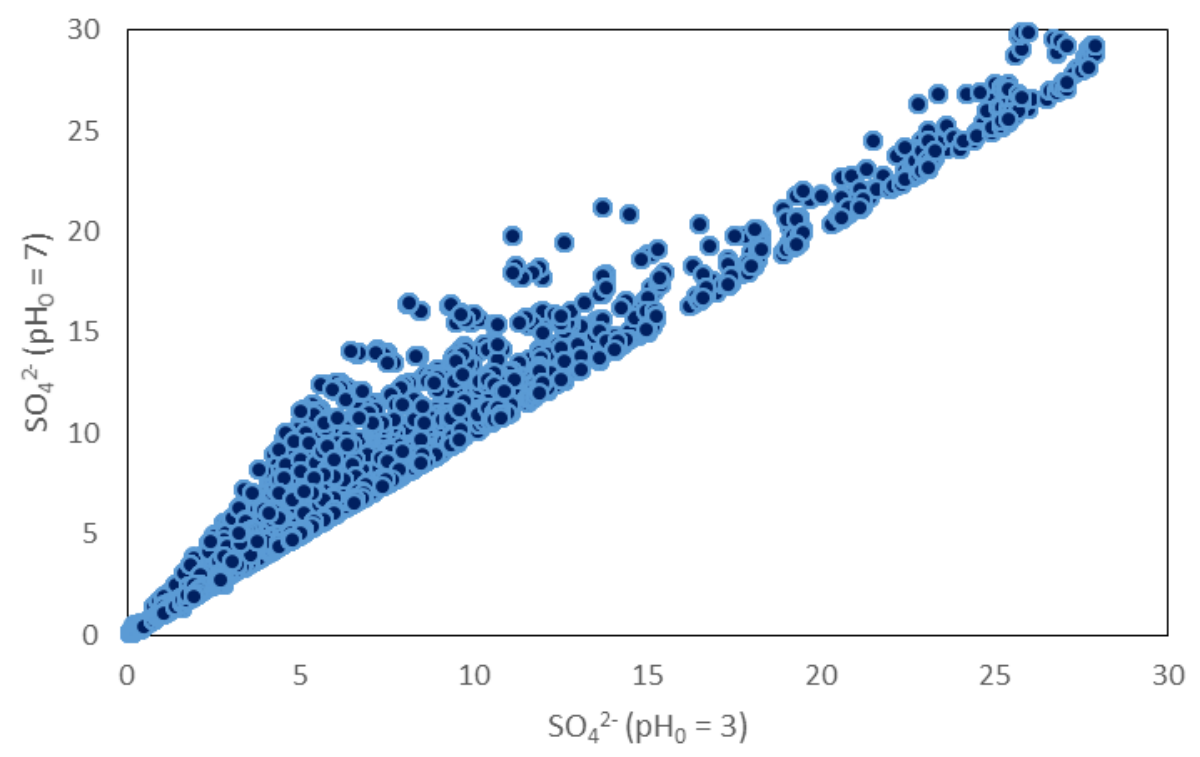

Figure S2: Predicted $\mathrm{SO}_{4}{ }^{2-}$ concentrations for AQCHEM-KMT for initial $\mathrm{pH}$ of 7 versus 3.

\section{References:}

5 Carlton, A.G., Bhave, P.V., Napelenok, S.L., Edney, E.O., Sarwar, G., Pinder, R.W., Pouliot, G.A., and Houyoux, M.: Model representation of secondary organic aerosol in CMAQv4.7, Environ. Sci. Technol., 44, 8553-8560, doi: 10.1021/es100636q, 2010.

Graedel, T.E. and Weschler, C.J.: Chemistry within aqueous atmospheric aerosols and raindrops, Reviews of Geophysics, 19(4), 505-539, doi:10.1029/RG019i004p00505, 1981.

10 Herrmann, H., Ervens, B., Jacobi, H.-W., Wolke, R., Nowacki, P., and Zellner, R.: A chemical aqueous phase radical mechanism for tropospheric chemistry, J. Atmos. Chem., 36, 231-284, doi:10.1023/A:1006318622743, 2000.

Lim, H., Carlton, A.G., and Turpin, B.J.: Isoprene forms secondary organic aerosol through cloud processing: model simulations, Environ. Sci. Technol., 39, 4441-4446, doi:10.1021/es048039h, 2005.

15 Pye, H.O.T., Pinder, R.W., Piletic, P.R., Xie, Y., Capps, S. L., Lin, Y., Surratt, J.D., Zhang, Z., Gold, A., Luecken, D.J., Hutzell, W.T., Jaoui, M., Offenberg, J.H., Kleindienst, T.E., Lewandoswki M., and Edney, E.O.: Epoxide pathways improve model predictions of isoprene markers and reveal key role of acidity in aerosol formation, Environ. Sci. Technol., 47, 1105611064, doi: 10.1021/es402106h, 2013.

Seinfeld, J. H. and Pandis, S. N.: Atmospheric Chemistry and Physics: From Air Pollution to Climate Change, 2nd Edn., John Wiley and Sons, Inc., 2006.

Warneck, P. and Williams, J.: The Atmospheric Chemist's Companion: Numerical Data for Use in the Atmospheric Sciences, Springer, 2012. 